\title{
Raltegravir, Tenofovir, ANd Emtricitabine in AN HIV-INFECTED Patient with HCV Chronic Hepatitis, NNRTI Intolerance and Protease InHibitors-INDUCED SEVERE Liver TOXICITY
}

\author{
F. Ortu 1 , L. E. Weimer 2 , M. Floridia ${ }^{2}$, P. E. Manconi ${ }^{1}$
}

\author{
${ }^{1}$ Clinic of Infectious Diseases, Department of Medicine and Immunology, University of Cagliari, Italy, \\ ${ }^{2}$ Department of Therapeutic Research and Medicines Evaluation, Istituto Superiore di Sanità, Rome, Italy
}

\begin{abstract}
Background: in HIV-infected patients with HCV-related chronic hepatitis, liver impairment and drug toxicity may substantially reduce the number of possible therapeutic options.

Case Description: we here describe the case of an HCV-HIV coinfected woman who had repeated severe episodes of drug-related liver toxicity with indinavir, saquinavir, fosamprenavir, and darunavir, with minimal further therapeutic options left in this class. Previous treatment-limiting side effects with efavirenz and nevirapine also precluded use of non-nucleoside reverse transcriptase inhibitors. Introduction of an integrase-inhibitor regimen based on raltegravir, tenofovir, and emtricitabine allowed a prompt achievement of undetectable viral load and a substantial rise of CD4 count to high levels, with no subsequent episodes of hepatic toxicity, and no other side effects.

Conclusions: given the relatively common prevalence of HCV-related chronic hepatitis among people with HIV, raltegravir might represent an important alternative option for a substantial number of patients who cannot be treated with protease inhibitors or NNRTI because of drug-related hepatic toxicity.
\end{abstract}

Key words: HIV/HCV, antiretroviral treatment, raltegravir, tenofovir, emtricitabine, chronic active hepatitis

\section{BACKGROUND}

HCV-infected patients who are also coinfected with HIV are at higher risk of progression of liver disease compared with patients infected with HCV alone [1]. In the case of chronic HCV infection, some antiretroviral drugs with potential hepatotoxicity should be avoided or be used with particular care. The risk of hepatic toxicity is particularly high for NNRTIs, which often cannot be prescribed safely because of a substantial risk of severe and sometimes fatal hepatic reactions [2,3]. Although less frequently, severe hepatic reactions may also be observed with protease inhibitors [4-7], and in such situations there may be limited therapeutic options left for an effective viral suppression. Integrase inhibitors represent innovative and promising drugs for patients who are intolerant or resistant to other classes of antiretroviral drugs [8-9], and are increasingly used in salvage regimens, with favourable immunological and virological responses. In the BENCHMRK studies, where HCV prevalence was about $10 \%$, the occurrence of grade 3-4 liver enzyme elevations was low (3.5-4.3\%), suggesting that raltegravir may be characterised by a favourable hepatic safety profile [10]. We here describe the case of an HCV-HIV coinfected woman with repeated episodes of severe liver toxicity attributable to protease inhibitors who was successfully switched to a regimen based on raltegravir, tenofovir and emtricitabine.

\section{Case Description}

Our patient, currently 43 years of age, born and living in Italy, was diagnosed with HCV in 1995 (positive for HCV IgG antibodies, HCV genotype 1A), at the age of 28, during a serological screening. Tests for HBV infection (HBV surface antigen, HBV surface and core antibody) were negative. In 1996, a diagnosis of HIV infection was made and the patient reported an history of previous intravenous drug use. She was clinically asymptomatic, with no history of past HIV-related symptoms, and a CD4 cell count of $318 / \mathrm{mm}^{3}$ at that time (CDC stage A2). A mild elevation of serum ALT concentrations (51 IU/l, reference range, 1-36 IU/l) was present. Before starting antiretroviral therapy, the patient had a first cycle of interferon treatment, administered three times per week for seven months, with no response to treatment. On March 1997 a combination regimen based on zidovudine plus didanosine was started, and on June 1998 this regimen was discontinued in order to introduce a PI-based HAART represented by zidovudine (ZDV), lamivudine (3TC) and indinavir. The treatment was effective in raising CD4 count (to $597 / \mathrm{mm}^{3}$ ) and decreasing viral load to undetectable levels within six months, but in September 1998 indinavir had to be replaced by saquinavir because of renal lithiasis, nausea, vomiting and ACTG grade 3 hepatic toxicity (ALT $248 \mathrm{U} / \mathrm{l}$ ). Treatment was maintained for about two years until the end of 2000, with limited adherence and development of resistance mutations to both reverse transcriptase $(41 \mathrm{~L}, 67 \mathrm{~N}$, $184 \mathrm{~V}, 215 \mathrm{Y}, 219 \mathrm{E})$ and HIV protease $(73 \mathrm{~S}, 90 \mathrm{M})$. From January 2001 to July 2002 different regimens based on NNRTI were given, but compliance remained low, virological response was limited (viral load undetectable in 2001, then rebounded to 2270 




Fig. 1. HIV RNA viral load, ALT/AST levels, CD4 cell count and antiretroviral treatment. ALT: Alanine aminotransferase; AST: Aspartate aminotransferase; TDF: tenofovir; FTC: emtricitabine; DRV/r: darunavir/ritonavir.

copies in 2002), and the patient developed side effects that required interruption of NNRTI treatment (first $\mathrm{d} 4 \mathrm{~T}+3 \mathrm{TC}+\mathrm{EFV}$, because of CNS symptoms, and subsequently $\mathrm{d} 4 \mathrm{~T}+\mathrm{ddI}+$ nevirapine, because of rash). In October 2002, a simplified regimen based on abacavir was started (d4T+ddI $+A B C)$. In April 2003, during treatment with this regimen, a genotypic resistance test was performed (viral load at the moment of the test: 2350 copies $/ \mathrm{ml}$ ), that substantially confirmed the mutation pattern observed two years before (RT: 41L, 67L, 184V, 215Y; PR: 73S, 90M). A liver biopsy was performed in May 2005. The results showed a general liver architecture altered by the presence of porto-portal septa and periterminal fibrosis, with inflammatory infiltration of portal spaces, piecemeal peripheral necrosis, and focal steatosis. A diagnosis of chronic hepatitis with moderate activity was made.

In September 2005 the patient started a new antiretroviral regimen based on abacavir, lamivudine and fosamprenavir/ritonavir. While the patient was on this regimen, she had two cycles of treatment with PEGinterferon alpha-2a plus ribavirine (800 $\mathrm{mg}$ per day) in July 2006 (administered for three months) and December 2006, respectively, with no response (HCV-RNA viral load in July 2007: 1,500,000 IU/ml). At the end of the second cycle the patient presented with asthenia, anorexia and hepatomegaly, and blood test revealed the presence of transaminase levels $>5 \mathrm{xUNL}$ (ACTG grade 3 toxicity). Antiretroviral treatment was discontinued, with regression of liver toxicity (ALT and AST levels of 108 and $87 \mathrm{U} / 1$, respectively, in January 2008). The reduced transaminase levels allowed the introduction of a new regimen based on tenofovir, emtricitabine and darunavir/ritonavir in March 2008. New blood tests in July 2008 revealed the presence of severe liver toxicity (ALT 515, AST 419, corresponding to ACTG grade 4 toxicity). This regimen was discontinued, with a prompt reduction of transaminase levels (ALT 109, AST 76 in October 2008). In October 2008, following a two-month period of treatment discontinuation, raltegravir was introduced (with tenofovir and emtricitabine). At this point (baseline for raltegravir treatment) the patient had a CD4 count of 542 cells $/ \mathrm{mm}^{3}$, an HIV-RNA viral load of 16380 copies $/ \mathrm{ml}\left(4.21 \log _{10} / \mathrm{ml}\right)$, and mildly increased ALT and AST levels (109 and $98 \mathrm{U} / 1$, respectively). After one month of treatment (November 2008) transaminase levels showed no increase (ALT 96, AST 76). In January 2009 CD4 cell count increased to $762 / \mathrm{mm}^{3}$ and viral load had became undetectable $(<50$ copies/ml, $\left.1.69 \log _{10}\right)$. ALT and AST levels were 146 and 128 , respectively. In the following evaluation, in April 2009, HIV-RNA remained undetectable, CD4 count increased to $927 / \mathrm{mm}^{3}$ and liver enzymes remained stable at relatively low levels (ALT 83, AST 67). Such data were substantially confirmed at two subsequent evaluations in July 2009 (CD4 926/ $\mathrm{mm}^{3}$, HIV-RNA $<50$ copies/ml, ALT 130, AST 105) and in September 2009 (CD4 956/ $\mathrm{mm}^{3}$, HIV-RNA <50 copies/ml, ALT 92, AST 114). During subsequent follow up, the patient is maintaining good clinical conditions and remains compliant with treatment.

\section{Discussion}

Liver toxicity represents a frequent drug-related complication in patients receiving antiretroviral treatment, and may commonly lead to interruption of treatment and / or hospital admission [11]. The risk of severe hepatic toxicity under antiretroviral treatment is increased in the presence of $\mathrm{HCV}$ coinfection $[6,12$, 13], and in such patients it is not always possible to assess the individual role of antiretroviral treatment and $\mathrm{HCV}$ coinfection in such events. The clinical history of our patient clearly indicated the presence of hepatic intolerance to protease inhibitors, with episodes of severe toxicity that promptly reversed following discontinuation of treatment. Such episodes occurred during treatment with different protease inhibitors, such as indinavir, saquinavir, fosamprenavir, and 
darunavir, leaving very limited therapeutic options in this class. Use of non-nucleoside reverse transcriptase was also precluded because of previous side effects with both efavirenz and nevirapine.

In this patient use of raltegravir allowed to obtain undetectable viral load and high CD4 levels, with no subsequent flares of hepatic toxicity, and no other relevant side effect. This favourable clinical course with a regimen that introduced an integrase inhibitor maintaining a nucleos $(\mathrm{t})$ ide backbone add further support to the hypothesis that previous episodes of liver toxicity were attributable to protease inhibitors and not to the nucleoside backbone concomitantly given to the patient. Some of the episodes occurred following anti$\mathrm{HCV}$ treatment, and interferon or ribavirine might also have played a facilitating role in the development of toxicity.

There is as yet limited information on the use of raltegravir in patients coinfected with $\mathrm{HCV}$, and further studies should evaluate its safety and efficacy in this particular population. Recent data by Vispo et al. suggest that, even if $\mathrm{HCV}$-coinfected patients receiving raltegravir may have an additional risk of liver toxicity compared to raltegravir-treated HIV monoinfected persons, this usually translates in mild liver enzyme elevations only, with no cases of grade 3-4 liver toxicity directly attributable to the drug [14]. Given the relatively common prevalence of HCV coinfection among people with HIV, raltegravir might represent an important alternative option for a substantial number of patients who cannot be treated with protease inhibitors or NNRTI because of hepatic intolerance.

Conflicts of interest: The authors do not have any commercial or other association that might pose a conflict of interest.

\section{REFERENCES}

1. Singal AK, Anand BS. Management of hepatitis $C$ virus infection in $\mathrm{HIV} / \mathrm{HCV}$ co-infected patients: clinical review. World J Gastroenterol. 2009;15:3713-24.

2. Vogel M, Rockstroh JK. Hepatotoxicity and liver disease in the context of HIV therapy. Curr Opin HIV AIDS. 2007;2:306-13.

3. Walker UA. Antiretroviral therapy-induced liver alterations. Curr Opin HIV AIDS. 2007;2:293-8.

4. Sulkowski MS, Mehta SH, Chaisson RE, Thomas DL, Moore RD. Hepatotoxicity associated with protease inhibitor-based antiretroviral regimens with or without concurrent ritonavir. AIDS. 2004 Nov 19;18(17):2277-84.

5. Pineda JA, Pérez-Elías MJ, Peña JM, Luque I, RodríguezAlcantara F. Fosamprenavir Expanded Access Program Group. Low rate of adverse hepatic events associated with fosamprenavir/ritonavir-based antiretroviral regimens. HIV Clin Trials. 2008; 9:309-13.

6. Jevtovic Dj, Ranin J, Salemovic D, et al. The prevalence and risk of hepatitis flares in a Serbian cohort of HIV and HCV co-infected patients treated with HAART. Biomed Pharmacother. 2008;62:21-5.
7. Pineda JA, Santos J, Rivero A, Abdel-Kader L, Palacios R, Camacho A, Lozano F, Macías J; Liverey Study Investigator Team. Liver toxicity of antiretroviral combinations including atazanavir/ritonavir in patients co-infected with HIV and hepatitis viruses: impact of pre-existing liver fibrosis. J Antimicrob Chemother. 2008;61(4):925-32.

8. Grinsztejn B, Nguyen BY, Katlama C, Gatell JM, Lazzarin A, Vittecoq D, et al. Safety and efficacy of the HIV1 integrase inhibitor raltegravir (MK-0518. in treatmentexperienced patients with multidrug-resistant virus: a phase II randomised controlled trial. Lancet. 2007; 369:1261-1269.

9. Croxtall JD, Keam SJ. Raltegravir: a review of its use in the management of HIV infection in treatment-experienced patients. Drugs. 2009;69:1059-75.

10. Steigbigel RT, Cooper DA, Kumar PN, Eron JE, Schechter M, Markowitz M, et al. Raltegravir with optimized background therapy for resistant HIV-1 infection. N Engl J Med. 2008; 24:339-54.

11. Núñez MJ, Martín-Carbonero L, Moreno V, Valencia E, Garcia-Samaniego J, Castillo JG, Barreiro P, GonzálezLahoz J, Soriano V. Impact of antiretroviral treatment-related toxicities on hospital admissions in HIV-infected patients. AIDS Res Hum Retroviruses. 2006;22:825-9.

12. Palacios R, Vergara S, Rivero A, Aguilar I, Macías J, Camacho A, Lozano F, García-Lázaro M, Pineda JA, TorreCisneros J, Márquez M, Santos J. Low incidence of severe liver events in HIV patients with and without hepatitis $C$ or B coinfection receiving lopinavir/ritonavir. HIV Clin Trials. 2006;7:319-23.

13. Meraviglia P, Schiavini M, Castagna A, Viganò P, Bini T, Landonio S, Danise A, Moioli MC, Angeli E, Bongiovanni M, Hasson H, Duca P, Cargnel A. Lopinavir/ritonavir treatment in HIV antiretroviral-experienced patients: evaluation of risk factors for liver enzyme elevation. HIV Med. 2004;5:334-43.

14. Vispo E, Mena A, Maida I, Blanco F, Cordoba M, Labarga $\mathrm{P}$, et al. Hepatic safety profile of raltegravir in HIV-infected patients with chronic hepatitis C. J Antimicrob Chemother. 2009 Dec 23. [Epub ahead of print]

Received: November 26, 2009 / Accepted: January 21, 2010

\author{
Address for correspondence: \\ Marco Floridia \\ Department of Therapeutic Research and \\ Medicines Evaluation \\ Istituto Superiore di Sanità \\ Viale Regina Elena 299 \\ 00161 Rome \\ Italy \\ Fax: $\quad+39.06 .4938 .7199$; \\ Phone: +39.06.4990.3228; \\ E-mail: marco.floridia@iss.it
}

